\title{
Genetic Diversity of Methicillin-
}

Resistant Staphylococcus Aureus Among Japanese Inpatients in the Early 1980s

Hui Zuo

Juntendo Daigaku Igakubu Daigakuin Igaku Kenkyuka

Yuki Uehara ( $\square$ yuuehara@juntendo.ac.jp)

Juntendo Daigaku https://orcid.org/0000-0003-1014-8888

Yujie Lu

Juntendo Daigaku Igakubu Daigakuin Igaku Kenkyuka

Takashi Sasaki

Sapporo Ika Daigaku

Keiichi Hiramatsu

Juntendo Daigaku Igakubu Daigakuin Igaku Kenkyuka

Research article

Keywords: Healthcare-associated methicillin-resistant Staphylococcus aureus (HA-MRSA), Japan, 1980s, tst-positive ST5-SCCmec II, imipenem

Posted Date: August 13th, 2020

DOI: https://doi.org/10.21203/rs.3.rs-46010/v1

License: (9) (1) This work is licensed under a Creative Commons Attribution 4.0 International License.

Read Full License 


\section{Abstract}

Background: Methicillin-resistant Staphylococcus aureus (MRSA) was recognized as a serious nosocomial pathogen in Japan starting in the early 1980s. Limited genotypic characteristics on healthcare-associated MRSA (HA-MRSA) associated with "hospital MRSA panics" in Japan in this era. Therefore, this study aimed to determine the characteristics of Japanese HA-MRSA strains in the early 1980s and their linkage with current MRSA strains.

Methods: For 194 S. aureus strains isolated in the early 1980s, we determined methicillin resistance phenotypically and genotypically and performed multilocus sequence typing (MLST), staphylococcal cassette chromosome mec ( $\mathrm{SCC} m e c)$ typing, and whole-genome sequencing.

Results: Twenty mecA-positive MRSA (10.3\%), 8 mecA-negative MRSA (4.1\%) and 3 mecA-positive methicillin-susceptible $S$. aureus (MSSA) (1.5\%) strains were identified. The frequent sequence type (ST) was ST30 $(n=11)$, followed by ST5 $(n=8)$, ST $81(n=4)$, and ST247 $(n=3)$. Rates of SCCmec types I, II, and IV were $65.2 \%, 13.0 \%$, and $17.4 \%$, respectively. ST30-SCCmec I $(n=7)$ and ST5-SCCmec I $(n=5)$ were predominant genotypes. Only two strains exhibited tst-positive ST5-SCCmec Il, which is the current Japanese HA-MRSA genotype. Moreover, $73.3 \%$ of SCCmec type I strains were susceptible to imipenem compared with SCCmec type II strains (0\%). All ST30 strains shared a common ancestor with strain $55 / 2053$, which resulted in a global pandemic of Panto-Valentine leukocidin (PVL)-positive and penicillinresistant MSSA spread in Europe and the United States in the 1950s.

Conclusions: Our results demonstrated the heterogeneous population structure of Japanese HA-MRSA during the early 1980s, which comprised diverse clones that are mostly rare in recent years. The shift to the current homogenous population structure of HA-MRSA strains consisting of tst-positive ST5-SCCmec II might result from the clinical introduction of new antimicrobials including imipenem.

\section{Background}

Staphylococcus aureus is a major opportunistic pathogen that can cause various life-threatening infections, and approximately $20 \%$ of healthy human individuals are persistent carriers of this bacterial species [1]. The first case of methicillin-resistant $S$. aureus (MRSA) was identified in the United Kingdom in 1961, just in one year after introduction of methicillin [2, 3]. Since then, MRSA remains a major clinical concern with both hospital-associated and community-associated MRSA (HA-MRSA and CA-MRSA, respectively) infections worldwide [4-6]. The number of deaths related to MRSA infection is still large and rivals HIV/AIDS infection in its public health impact [7].

Nasal carriage of bacteria including $S$. aureus in human hosts can serve as a reservoir for the spread of the pathogen and can predispose the host to subsequent infections [8-11]. MRSA infections are often resistant to empirical antimicrobial therapy because of multidrug-resistance including $\beta$-lactam antimicrobials $[12,13]$. Therefore, nasal swabbing has been performed for MRSA screening as an infection control measure $[14,15]$. 
MRSA is generated when methicillin-susceptible $S$. aureus (MSSA) acquires the exogenous mecA gene encoding the penicillin-binding protein 2' (PBP2'), which is located on a mobile genetic element named as staphylococcal cassette chromosome mec (SCCmec) [16]. According to the International Working Group on the Classification of Staphylococcal Cassette Chromosome Elements, strains can be classified by SCCmectypes (I-XIV) based on the combinations of mec and $c c r$ gene complexes [6, 17-19]. Each MRSA clone has been described by the combination of the chromosomal genotype of the recipient MSSA strain and the genotype of the integrated SCCmec. Therefore, multilocus sequence typing (MLST) and SCCmec typing by PCR and/or sequencing methods have been widely used as gold standard methods in molecular epidemiological studies of MRSA [4, 20-23]. In addition, whole-genome sequencing (WGS) of bacterial isolates by next-generation sequencing (NGS) technologies has recently become a promising tool for molecular typing. WGS by NGS has made remarkable advances for microbiologists; these changes reflect decreased costs, increased capabilities of data processing systems, and ever-larger databases of NGS datasets [24]. However, these molecular typing tools for MRSA isolates were established in the 2000s; thus, there have been few reports on molecular epidemiology of MRSA strains before then.

Population structure tracking of bacteria over time can provide crucial insights into infection control and appropriate use of antimicrobials in clinical practice [25]. Therefore, the aims of this study were to retrospectively review the population structure of Japanese HA-MRSA strains isolated in the early 1980s using MLST, SCCmec typing and phylogenetic analysis based on whole-genome single nucleotide polymorphisms (SNPs), and to compare the population structure with that of strains isolated in recent years. Consequently, we report the polyclonal population structure of Japanese HA-MRSA in the 1980s, which were composed of rare clones in recent years.

\section{Methods}

\section{Bacterial isolates collection}

This study examined a collection of 194 S. aureus strains (designated as "N" strains) that were isolated from 184 Japanese inpatients in 22 prefectures between January 1982 and December 1983. The isolates were mainly from Fukushima (19, 9.8\%), Miyagi (18, 9.3\%), Okinawa (18, 9.3\%), and Osaka (18, 9.3\%). Specimen types were as follows: pus $(88,45 \%)$, sputum $(43,22 \%)$, blood $(20,10 \%)$, urine $(15,8 \%)$, pharynx $(10,5 \%)$, other $(7,4 \%)$, and unknown $(12,6 \%)$, suggesting that skin and soft tissue infection and respiratory tract infection were major infectious diseases in the study population. A subset of these $\mathrm{N}$ strains was previously reported [26]. Catalase-positive, Gram-positive cocci that were presumptively identified as staphylococci by colonial morphology were subcultured on trypticase soy agar (BD Japan, Co., Ltd., Tokyo, Japan). Tube coagulase tests with rabbit plasma (Denka Seiken Co., Ltd., Tokyo, Japan) were performed, and only coagulase-positive staphylococcal strains were selected for further investigation. S. aureus was confirmed by a PCR method targeting the thermonuclease (nuc) gene after DNA extraction [27]. 


\section{DNA extraction}

A single colony of each strain was used for bacterial genomic DNA extraction by using the QIAamp ${ }^{\circledR}$ DNA Mini Kit (QIAGEN, Hilden, Germany). Purified genomic DNA was used for PCRs and sequencingbased methods.

\section{Determination of methicillin-resistance}

We determined methicillin-resistance in all $S$. aureus strains by evaluating oxacillin and cefoxitin susceptibilities according to Clinical and Laboratory Standards Institute (CLSI) M100-S22 performance standards [28]. In addition, all strains were genetically assessed by two different PCRs for the presence of the $m e c A$ gene $[29,30]$.

\section{Antimicrobial susceptibility testing}

Minimum inhibitory concentration (MIC) tests for other antimicrobial agents were performed by the broth microdilution method by cation-adjusted Mueller-Hinton broth (CAMHB) using the Dry plate "Eiken" DP32 (Eiken Chemical Co., Tokyo, Japan), containing oxacillin, cefoxitin, ampicillin, cefazolin, cefmetazole, flomoxef, imipenem, gentamicin, arbekacin, minocycline, erythromycin, clindamycin, levofloxacin, vancomycin, teicoplanin, linezolid, fosfomycin and trimethoprim-sulfamethoxazole. For oxacillin and cefoxitin, agar dilution method was performed using Mueller Hinton II agar (Becton, Dickinson and Co.) with $2 \% \mathrm{NaCl}$. MICs were examined by visual observation and interpreted according to CLSI M100-S22 performance standards [28]. Differences in the rates of Imipenem susceptibility rates by SCCmec types were evaluated using the Fisher's exact test.

\section{Molecular typing of MRSA strains}

SCCmec typing (I-V) was performed by a multiplex PCR method reported previously [22]. spa-typing and multilocus sequence typing (MLST) were carried out as previously reported [31-33]. Eleven exotoxin genes, encoding staphylococcal enterotoxins SEA (sea), SEB (seb), SEC (sec), SED (sed), SEE (see), SEG (seg), SEH (seh), SEI (sei), SEJ (sei); exfoliative toxin A, B (ETA; eta, ETB; etb, respectively); TSST-1 (tst); and Panton-Valentine leukocidin (PVL; lukS and lukF) were detected by PCR as reported previously [3436].

\section{WGS and additional molecular phylogenetic analysis}

The Nextera XT DNA sample preparation kit (Illumina Inc., San Diego, CA, USA) was used for sample preparation for WGS. Sequencing was performed using a paired-end $2 \times 250$ or 300-bp cycle run on the Illumina MiSeq sequencing system using MiSeq reagent kit v2 or v3 (Illumina Inc.).

De novo assembly was performed using the CLC Genomics Workbench version 9 (Qiagen N.V., Venlo, The Netherlands). Assembled contigs were submitted to spaTyper 1.0 for spa-typing, ResFinder 3.0 for detection of acquired drug-resistant genes, and MLST 1.8 for MLST, which are all housed on the Center for Genomic Epidemiology (CGE) website (http://www.genomicepidemiology.org//) [24, 37-39]. 
Assembled contigs of strains isolated in this study were also submitted to CSI phylogeny 1.4 on the CGE website to call SNPs and infer the phylogenetic relationship among strains described in this study and the 125 complete genome sequenced-S. aureus strains, which were available from the National Center for Biochemistry Information (NCBI) database [40]. In addition, pairwise SNP analyses were performed focusing on clonal complexes (CC) 5 and CC30 strains in order to elucidate relatedness with and preservation among the recent MRSA strains.

\section{Results}

\section{Rates of MRSA and MSSA strains in Japanese hospitals during the early 1980s}

In total, 194 isolates were identified as $S$. aureus. One hundred and seventy-four patients yielded one strain per each patient, whereas ten patients yielded two strains per each patient. According to phenotypic and genotypic determination of methicillin-resistance, we identified 20 mecA-positive MRSA (10.3\%), 8 mecA-negative MRSA (4.1\%), 3 mecA-positive MSSA (1.5\%) and 163 mecA-negative MSSA (84.0\%) strains. Consequently, the phenotypic methicillin-resistant rate of the strains described in this study was $14.4 \%$ (28 of 194 strains).

\section{Antimicrobial susceptibilities in MRSA and MSSA strains from Japanese inpatients during the early 1980s}

As shown in Table 1, all S. aureus strains described in this study were susceptible to levofloxacin, which had been not launched in Japan in the 1980s. All S. aureus strains were susceptible to anti-MRSA agents including vancomycin, teicoplanin, linezolid, and arbekacin, irrespective of methicillin resistance. Moreover, $32.1 \%$ of MRSA strains (9 of 28 strains) showed resistance to imipenem, which was unavailable in Japan during the study period, whereas all MSSA strains were susceptible to imipenem. 
Table 1

Minimum inhibitory concentrations of antimicrobial agents against methicillin-resistant and methicillinsusceptible Staphylococcus aureus isolates.

\begin{tabular}{|c|c|c|c|c|c|c|}
\hline \multirow[t]{3}{*}{ Antimicrobial agents $^{b}$} & \multicolumn{6}{|c|}{$\operatorname{MICs}(\mathrm{mg} / \mathrm{L})^{\mathrm{c}}$} \\
\hline & \multicolumn{3}{|c|}{$\operatorname{MRSA}^{c}(n=28)$} & \multicolumn{3}{|c|}{$\operatorname{MSSA}^{c}(n=166)$} \\
\hline & Range & $\mathrm{MIC}_{50}$ & $\mathrm{MIC}_{90}{ }_{90}$ & Range & $\mathrm{MIC}_{50}$ & $\mathrm{MIC}_{90}$ \\
\hline Oxacillin & $4-256$ & 32 & 128 & $\leq 0.12-2$ & 0.25 & 1 \\
\hline Cefoxitin & $4-256$ & 16 & 64 & $\leq 4-4$ & $\leq 4$ & $\leq 4$ \\
\hline Ampicillin & $\begin{array}{l}0.25- \\
>16\end{array}$ & $>16$ & $>16$ & $\leq 0.12->16$ & 4 & 16 \\
\hline Cefazolin & $\begin{array}{l}\leq 0.5- \\
>16\end{array}$ & $>16$ & $>16$ & $\leq 0.5-2$ & $\leq 0.5$ & 1 \\
\hline Cefmetazole & $\leq 1->32$ & 16 & 32 & $\leq 1-4$ & $\leq 1$ & 2 \\
\hline Flomoxef & $\begin{array}{l}\leq 0.5- \\
>16\end{array}$ & 4 & $>16$ & $\leq 0.5-2$ & $\leq 0.5$ & $\leq 0.5$ \\
\hline Imipenem & $\underset{>8}{\leq 0.25-}$ & 2 & $>8$ & $\leq 0.25$ & $\leq 0.25$ & $\leq 0.25$ \\
\hline Gentamicin & $\underset{>8}{\leq 0.25-}$ & $>8$ & $>8$ & $\leq 0.25->8$ & $\leq 0.25$ & 1 \\
\hline Arbekacin & $\leq 0.25-$ & 1 & 4 & $\leq 0.25-8$ & 0.5 & 1 \\
\hline Minocycline & $\leq 2->8$ & $\leq 2$ & 8 & $\leq 2$ & $\leq 2$ & $\leq 2$ \\
\hline Erythromycin & $\begin{array}{l}\leq 0.12- \\
>4\end{array}$ & $>4$ & $>4$ & $\leq 0.12->4$ & 0.5 & $>4$ \\
\hline Clindamycin & $\begin{array}{l}\leq 0.06- \\
>2\end{array}$ & 0.12 & $>2$ & $\leq 0.06->2$ & 0.12 & 0.25 \\
\hline Levofloxacin & $\leq 0.25-$ & 0.5 & 0.5 & $\leq 0.25-2$ & 0.5 & 0.5 \\
\hline Vancomycin & $\leq 0.5-2$ & 1 & 1 & $\leq 0.5-2$ & 1 & 1 \\
\hline
\end{tabular}

${ }^{a} \mathrm{MIC}_{50} / \mathrm{MIC}_{90}$, MIC required to inhibit the growth of $50 \%$ or $90 \%$ of the strains, respectively.

${ }^{\mathrm{b}}$ MICs of oxacilllin and cefoxitin were determined by the agar dilution method; all other MICs were determined by the broth microdilution method.

c MRSA, methicillin-resistant Staphylococcus aureus; MSSA, methicillin-susceptible Staphylococcus aureus, MIC, minimum inhibitory concentration. 


\begin{tabular}{|c|c|c|c|c|c|c|}
\hline \multirow[t]{3}{*}{ Antimicrobial agents ${ }^{b}$} & \multicolumn{6}{|c|}{ MICs (mg/L)c } \\
\hline & \multicolumn{3}{|c|}{$\operatorname{MRSA}^{c}(n=28)$} & \multicolumn{3}{|c|}{$\operatorname{MSSA}^{c}(n=166)$} \\
\hline & Range & $\mathrm{MIC}_{50}$ & $\mathrm{MIC}_{90}{ }_{90}$ & Range & $\mathrm{MIC}_{50}$ & $\mathrm{MIC}_{90}$ \\
\hline Teicoplanin & $\leq 0.5-2$ & $\leq 0.5$ & 1 & $\leq 0.5-2$ & $\leq 0.5$ & 1 \\
\hline Linezolid & $0.5-2$ & 1 & 2 & $0.5-4$ & 2 & 2 \\
\hline Fosfomycin & $\begin{array}{l}\leq 32- \\
>128\end{array}$ & $\leq 32$ & $>128$ & $\leq 32->128$ & $\leq 32$ & $\leq 32$ \\
\hline $\begin{array}{l}\text { Sulfamethoxazole/ } \\
\text { trimethoprim }\end{array}$ & $\leq .5 / 0.5$ & $\leq .5 / 0.5$ & $\leq .5 / 0.5$ & $\begin{array}{l}\leq 9.5 / 0.5- \\
>38 / 2\end{array}$ & $\leq .5 / 0.5$ & $\leq .5 / 0.5$ \\
\hline \multicolumn{7}{|c|}{${ }^{a} \mathrm{MIC}_{50} / \mathrm{MIC}_{90}, \mathrm{MIC}$ required to inhibit the growth of $50 \%$ or $90 \%$ of the strains, respectively. } \\
\hline \multicolumn{7}{|c|}{$\begin{array}{l}\text { b MICs of oxacilllin and cefoxitin were determined by the agar dilution method; all other MICs were } \\
\text { determined by the broth microdilution method. }\end{array}$} \\
\hline
\end{tabular}

\section{Detailed genetic characterizations of MRSA and mecA-positive MSSA strains in Japan during the early 1980s}

In order to understand the detailed characteristics of Japanese HA-MRSA strains during the early 1980s, we performed genetic characterizations using MLST, SCCmec typing, spa-typing, toxin profiling and acquired antimicrobial resistance gene profiling (Table 2, Additional_file_2).

Among 31 strains in total including phenotypically-identified MRSA and mecA-positive MSSA, the most frequent sequence type (ST) was ST30 $(n=11,35.5 \%)$, followed by ST5 $(n=8,25.8 \%)$, ST81 $(n=4$, $12.9 \%$ ) and ST247 ( $n=3,9.7 \%)$. SCCmec types I, II, and IV were found in $65.2 \%, 13.0 \%$, and $17.4 \%$ among 23 mecA-positive strains, respectively. ST30-SCCmec I $(n=7)$ was the most predominant genotype, followed by ST5-SCCmec I $(n=5)$, ST30-SCCmec IV $(n=4)$, ST247-SCCmec I $(n=3)$ and ST5-SCCmec II $(\mathrm{n}=2)$. The genotype exhibiting tst-positive ST5-SCCmec Il, which was known as the current Japanese HA-MRSA genotype, was identified in only two strains, N106 and N315. Eight PVL-positive strains were identified, all of which were ST30.

Among acquired antimicrobial resistance genes detected in this study, mecA was the most frequent $(\mathrm{n}=$ $23)$, followed by blaZ $(\mathrm{n}=22)$, ermA $(\mathrm{n}=19)$, ant $(9)$-la $(\mathrm{n}=19) \square \operatorname{aac}\left(6^{\prime}\right)-\operatorname{aph}\left(2^{\prime \prime}\right)(\mathrm{n}=18)$. All mecA-positive strains had aminoglycoside resistance genes, and multiple strains carried resistance genes related to macrolide (82.6\%), tetracycline (39.1\%), and phenicol (13.0\%). 
Consequently, the prevalence of various MRSA clones during the early 1980s was found in this study, and diverse genotypes were detected even among MRSA strains exhibiting the same ST. These results suggested that the population structure of Japanese HA-MRSA strains during the early 1980s had been notably different from the monoclonal population composed of ST5-SCCmec II known as HA-MRSA in recent years.

\section{Antibiograms of the strains by SCC mec types}

In order to consider the mechanisms for shift of population structure of Japanese HA-MRSA strains from polyclonal during the early 1980 s to monoclonal in recent years, we compared the antibiograms of MRSA strains across SCCmec types (Table 3, Additional_file_3).

Strains that carried SCCmectypes I and II were highly resistant to $\beta$-lactams including oxacillin, however, those that carried SCCmec type IV were more susceptible to $\beta$-lactams despite being mecA-positive. Among 15 strains carrying SCCmec type I, the rate of erythromycin resistance was the highest $(80.0 \%)$, followed by resistance to gentamycin (66.7\%), clindamycin (40.0\%), and minocycline (26.7\%), which were commonly used antimicrobials at that time. Imipenem susceptibility rates in SCCmec type I strains were $73.3 \%$ ( 11 of 15 strains) as compared with $0 \%$ in SCC mec type II strains ( 0 of 3 strains) $(p<0.05)$.

The mecA-negative MRSA strains $(\mathrm{n}=8)$ were more susceptible to $\beta$-lactams, and especially, all strains were susceptible to imipenem except one isolate (N89). Lower MICs of these strains for aminoglycosides, minocycline, erythromycin and clindamycin were observed compared with the mecA-positive strains.

\section{Population structure of MRSA and mecA-positive MSSA strains in Japan during the early 1980 s}

In order to understand the population structure of Japanese HA-MRSA strains during the 1980s, we performed a phylogenetic analysis based on whole-genome SNPs for 20 mecA-positive MRSA, 8 mecAnegative MRSA, 3 mecA-positive MSSA, and 125 reference S. aureus strains (Fig. 1).

The neighbor-joining (NJ) tree was constructed by alignment of 41,910 SNP sites. The percentage of reference genome (Staphylococcus argenteus MSHR1132) covered by all isolates was $89.27 \%(1,739,038$ of $2,762,785$ positions). Strains belonging to the CC30 cluster were most predominant $(n=11)$, followed by CC5 $(n=9), \operatorname{CC} 1(n=4)$, and CC8 $(n=4)$, indicating that the population structure of MRSA strains during the early 1980 s was composed of diverse clones.

\section{Whole-genome SNP analysis of CC30 and CC5 strains}

In order to elucidate whether prevalence of MRSA strains in this study resulted from a recent transmission event, the detailed pairwise SNPs analyses in 11 ST30 and 9 CC5 strains described in this study were performed, respectively (Figs. 2A and 2B).

The detailed pairwise SNPs analysis of 11 ST30 strains described in this study and 6 CC30 reference strains was performed (Fig. 2A). The percentage of reference genome (SJTUF_J27, ST433) covered by all strains was $89.16 \%(2,500,756$ of $2,804,761$ positions $)$ in the SNPs analysis. SNP differences ranged 
from 28 to 1008. All CC30 strains described in this study clustered into a single clade and were most closely related to the MSSA strain 55/2053 isolated in the United Kingdom in 1955 [41]. N83 and N86 strains, which were isolated in Kumamoto in the same year and exhibited the same spa-type t1504, showed 28 SNP differences, suggesting a direct horizontal spread in this hospital. However, SCCmectype and antimicrobial resistance gene profiles differed between these two strains, suggesting that these strains were independently acquired by each inpatient from different infectious sources. Consequently, the ST30 strains isolated in this study could be recognized as causing a regional outbreak, with the number of SNPs ranging from 28 to $164[42,43]$.

Next, we performed SNP analysis among 9 CC5 MRSA strains in this study and 32 reference strains (Fig. 2B). The percentage of reference genome (ED98) covered by all strains was $89.13 \%(2,517,393$ of $2,824,404$ positions) in the SNP analysis. SNP differences ranged from 0 to 621 . ST5 MRSA strains described in this study clustered into five different clades. Strains N366, N98, N345, N279, and N283 harboring SCCmec type I belonged to a single cluster, and SNP differences ranged from 30 to 84. N279 and N283 strains, which were isolated in Miyagi in the same year, showed only 30 SNP differences and similar profiles regarding spa type and toxin and antimicrobial resistance genes, suggesting a recent transmission event. Although N366 and N98 strains only exhibited 41 SNP differences, these strains were isolated in a different cross-strait region (Table 2, Additonal_file_2). According to these results, strains exhibiting ST5-SCC mec I belonging to this clade could be recognized as causing a regional outbreak, as contrasted with strains $16125,18412,18341,10388,15532,18583,16035$, and 10497, which were recovered with the number of SNPs ranging from 0 to 7 at a hospital during the outbreaks in Switzerland (Fig. 2B) [44].

Thus, our results suggested that MRSA clones exhibiting ST30- and ST5-SCCmec I had been spread as regional clones throughout Japan in the early 1980s.

\section{Discussion}

This study indicated that the population of Japanese HA-MRSA strains present during the early $1980 \mathrm{~s}$ showed a polyclonal structure composed of multiple clones, most of which have become uncommon recently. The recent monoclonal population structure of HA-MRSA strains in Japan likely formed over the past several decades, possibly in response to the release of various new antimicrobial agents and changes in MRSA treatment strategies from the 1980s onward.

This study indicated that PVL-positive ST30-SCCmec I was the most frequent genotype among Japanese HA-MRSA strains in the early 1980s. According to a previous report, nosocomial outbreaks of MRSA exhibiting PVL-positive ST30-SCCmec IV had frequently occurred in the late 1980s and early 1990 s in Japanese hospitals [45], whereas this genotype comprised a minor population in the early 1980s in this study. Our results suggested that the population structure of Japanese HA-MRSA strains had been dynamically replaced through the 1980s. This dynamic replacement largely due to ST30 MRSA clones might have resulted from high genetic and phenotypic diversity among ST30 MRSA strains. Indeed, in 
addition to mecA-positive MRSA, mecA-negative MRSA and mecA-positive MSSA, which were rarely isolated in recent years, were also found among ST30 strains in this study. Differences in observed $\mathrm{SCCmec}$ types and presence or absence of PVL genes were also identified among these strains.

As previously reported, the CC30 S. aureus lineage can be divided into three clusters: Clade 1 (representative strain 55/2053; PVL-positive and penicillin-resistant MSSA), Clade 2 (representative strain TCH60; PVL-positive CA-MRSA harboring SCCmec type IV), and Clade 3 (representative strain MRSA252/EMRSA-16; PVL-negative HA-MRSA harboring SCCmec type II or IV) [41]. Strains in Clade 1 cause severe infections and had been the epidemic clone in Europe, the United States, and Australia in the 1950s [46-50]. The percent of $S$. aureus infections caused by Clade 1 strains decreased dramatically by the mid-1960s, resulting from methicillin use for the treatment of penicillin-resistant strains [51]. However, this clone had re-emerged as HA-MRSA in Japanese hospitals in the early 1980s according to our results. Our phylogenetic analysis based on whole-genome SNPs demonstrated that all Japanese ST30 strains clustered into a single clade including strain 55/2053, suggesting that a Clade 1 strain imported from overseas had acquired SCCmec type I, SCCmec type IV, or unknown genetic factors and had already increased diversity in Japanese hospitals in the early 1980s. Consequently, the ST30 strains had likely spread throughout Japan as a nosocomial clone causing a regional outbreak at that time.

We previously reported that strains harboring SCCmec type II accounted for a large portion of MRSA in Japanese hospitals in the late 1990s [20,52]. The polyclonal population structure of MRSA strains in the early 1980 s observed in this study had been replaced by a monoclonal population structure largely composed of the current Japanese HA-MRSA clone since the late 1990s, coinciding with the new release of various antimicrobial agents such as third-generation cephalosporins, carbapenems and fluoroquinolones from the 1980s onward. The treatment of MRSA infections had been dramatically altered at those times. Imipenem was frequently used for the treatment of MRSA infections before the clinical introduction of vancomycin in 1991 in Japan. Strains harboring SCCmectype I, which was the predominant genotype in this study and some countries including the United Kingdom in the early 1980s [20,53], displayed high rate of imipenem-susceptibility, as contrasted with SCCmec II strains. Imipenem/cilastatin was launched in 1987 and had been used as an anti-MRSA agent before the clinical introduction of vancomycin in 1991 in Japan. According to the data in Japanese hospitals, the simple selective pressure induced by imipenem, might have caused dynamic population shift from the imipenem-susceptible diverse MRSA clones to the imipenem-resistant ST5-SCCmec II MRSA between 1980 and 2000. Also, in vitro exposure to imipenem can induce the conversions of heterogeneous-tohomogeneous and Eagle type-to-homogeneous methicillin resistance in $S$. aureus strains via chromosomal gene mutations such as $\operatorname{vraSR}$ and $r p o B$ genes $[6,21,54-58]$. Thus, the clinical use of imipenem against MRSA infections might have contributed to the attenuation of MRSA strains with atypical methicillin-resistance phenotype, which were rarely isolated in recent years.

In the current study, multiple MRSA strains exhibiting ST247-SCCmec I were isolated in the northeast area in Japan. This genotype is known as the Iberian clone, and had been dominated internationally up to the early 2000s [59-62]. Our results showed the local existence of the Iberian clone in Japan during the early 
1980s. Interestingly, all of ST247-SCCmec I strains in this study were resistant to imipenem. At the start of clinical use of imipenem around the world, the Iberian clone might have been spread from the 1990s to the early 2000s. The Iberian clone has been already replaced by the current major epidemic clones [63].

This study showed that ST5-SCCmec I was the second most frequent genotype among Japanese HAMRSA strains in the early 1980s. This genotype is shared by EMRSA-3, which was the most common MRSA clone in the United Kingdom in 1987-1988 along with EMRSA-15 (ST22-SCCmec IV) and EMRSA16 (ST36-SCCmec II) [4]. Studies conducted in South America in the late 1990s have identified the Cordobes/Chilean clone, which is genetically related to the EMRSA-3 but presents differences in the pulsed-field gel electrophoresis (PFGE) pattern and spa type [64-66]. This MRSA clone was also detected at a high rate in hospitals in South Brazil in 2008, suggesting the potential for re-dissemination in Brazil $[67,68]$. Although this MRSA clone exhibiting ST5-SCCmec I has remained uncommon in regions outside of South America in recent years, continuous monitoring is needed to prevent future outbreaks.

In 2001, we reported on the complete genome sequence of the $S$. aureus strain N315 with ST5-SCCmec II, as a representative strain of New York/Japan HA-MRSA clone [69]. The strain N315 was isolated from the pharyngeal smear of a Japanese patient in 1982, and also described in this study. Our results showed that the Japanese HA-MRSA clone exhibiting genotype of the strain N315 with multi-drug resistance had already been existed as a minor clone in the early 1980s, and could be the most frequent HA-MRSA in the 1990s and 2000s due to wide use of new antimicrobials. Whereas in the 2010s, awareness of appropriate use of antimicrobials has been raised to combat antimicrobial resistance issues in the world. It was reported that the population of Japanese HA-MRSA had been shifting again from N315-like CC5-SCCmec II to CC8-SCCmec IV and CC1-SCC mec IV, both of which had higher susceptibility to cefotaxime, levofloxacin, clarithromycin and clindamycin in the 2010s [70]. The history of the clonal change of MRSA in decades seems to reflect the recent appropriateness of our antimicrobial usage.

\section{Conclusions}

In conclusion, this study revealed the alteration of population structures of HA-MRSA strains from the early 1980 s onward as a consequence of elimination by new drug developments over the past several decades. Our findings will contribute to future progress of infection control measurements and appropriate use of antimicrobials for nosocomial infections caused by MRSA.

\section{Abbreviations}

\section{CA-MRSA}

community-associated methicillin-resistant Staphylococcus aureus, CAMHB:cation-adjusted MuellerHinton broth; CLSI:Clinical and Laboratory Standards Institute; CC:clonal complex; CGE:Center for Genomic Epidemiology; HA-MRSA:healthcare-associated methicillin-resistant Staphylococcus aureus; MSSA, methicillin-susceptible Staphylococcus aureus; MIC:minimum inhibitory concentration; MLST:multilocus sequence typing; NGS:next-generation sequencing; NCBI:National Center for 
Biochemistry Information; NJ, neighbor-joining; NT, non-typable; PCR:polymerase chain reaction;

PBP2':penicillin-binding protein 2'; PVL:Panton-Valentine leucocidin; PFGE:pulsed-field gel electrophoresis; SCCmec:Staphylococcal cassette chromosome mec, SNPs:single nucleotide polymorphisms;

ST:sequence type; WGS:whole-genome sequencing.

\section{Declarations}

\section{Ethics approval and consent to participate}

The administrative office of the committee of ethics of Juntendo University Faculty of Medicine reviewed this study and permitted to waive the official approval of the committee and the informed consent, due to the retrospective nature of the research using the isolates without any personal information of patients.

\section{Consent for publication}

Not applicable.

\section{Availability of data and materials}

The read data for whole-genome sequencing analysis of strains in this study have been deposited in GenBank under accession number DRA010146. Other data and materials in this study are included within the article.

\section{Competing interests}

The authors declare that they have no competing interests.

\section{Funding}

This study was supported by a Grant-in-Aid (S1201013) from the Ministry of Education, Culture, Sports, Science, and Technology Supported Program for the Strategic Research Foundation at Private Universities (2012-2016). The funder had no role in study design, data collection, and interpretation or the decision to submit the work for publication.

\section{Authors' contributions}

$\mathrm{HZ}, \mathrm{YU}$ and $\mathrm{KH}$ participated in the study design; $\mathrm{HZ}, \mathrm{YU}$ and $\mathrm{KH}$ collected and analyzed clinical information; $\mathrm{HZ}, \mathrm{YU}, \mathrm{YL}, \mathrm{TS}$ and $\mathrm{KH}$ carried out all microbiological experiments and data analysis; $\mathrm{HZ}, \mathrm{YU}$, TS and $\mathrm{KH}$ drafted and revised the manuscript. All authors read and approved the final manuscript.

\section{Acknowledgements}

Not applicable. 


\section{References}

1. Kluytmans J, van Belkum A, Verbrugh H. Nasal carriage of Staphylococcus aureus. epidemiology, underlying mechanisms, and associated risks. Clin Microbiol Rev. 1997;10(3):505-20.

2. Jevons MP, Coe AW, Parker MT. Methicillin resistance in staphylococci. Lancet. 1963;1(7287):904-7.

3. Barber M. Methicillin-resistant staphylococci. J Clin Pathol. 1961;14:385-93.

4. Enright MC, Robinson DA, Randle G, Feil EJ, Grundmann H, Spratt BG. The evolutionary history of methicillin-resistant Staphylococcus aureus (MRSA). Proc Natl Acad Sci U S A. 2002;99(11):768792.

5. Gorak EJ, Yamada SM, Brown JD. Community-acquired methicillin-resistant Staphylococcus aureus in hospitalized adults and children without known risk factors. Clin Infect Dis. 1999;29(4):797-800.

6. Hiramatsu K, Ito T, Tsubakishita S, Sasaki T, Takeuchi F, Morimoto Y, et al. Genomic Basis for Methicillin Resistance in Staphylococcus aureus. Infect Chemother. 2013;45(2):117-36.

7. Boucher HW, Corey GR. Epidemiology of methicillin-resistant Staphylococcus aureus. Clin Infect Dis. 2008;46(Suppl 5):344-9.

8. Sivaraman K, Venkataraman N, Cole AM. Staphylococcus aureus nasal carriage and its contributing factors. Future Microbiol. 2009;4(8):999-1008.

9. von Eiff C, Becker K, Machka K, Stammer H, Peters G. Nasal carriage as a source of Staphylococcus aureus bacteremia. Study Group. N Engl J Med. 2001;344(1):11-6.

10. Wertheim HF, Melles DC, Vos MC, van Leeuwen W, van Belkum A, Verbrugh HA, et al. The role of nasal carriage in Staphylococcus aureus infections. Lancet Infect Dis. 2005;5(12):751-62.

11. Mulcahy ME, McLoughlin RM. Host-bacterial crosstalk determines Staphylococcus aureus nasal colonization. Trends Microbiol. 2016;24(11):872-86.

12. Hartman BJ, Tomasz A. Low-affinity penicillin-binding protein associated with beta-lactam resistance in Staphylococcus aureus. J Bacteriol. 1984;158(2):513-6.

13. Utsui Y, Yokota T. Role of an altered penicillin-binding protein in methicillin- and cephem-resistant Staphylococcus aureus. Antimicrob Agents Chemother. 1985;28(3):397-403.

14. Coello R, Jimenez J, Garcia M, Arroyo P, Minguez D, Fernandez C, et al. Prospective study of infection, colonization and carriage of methicillin-resistant Staphylococcus aureus in an outbreak affecting 990 patients. Eur J Clin Microbiol Infect Dis. 1994;13(1):74-81.

15. El-Bouri K, El-Bouri W. Screening cultures for detection of methicillin-resistant Staphylococcus aureus in a population at high risk for MRSA colonisation: identification of optimal combinations of anatomical sites. Libyan J Med. 2013;8:22755.

16. Ito T, Katayama Y, Hiramatsu K. Cloning and nucleotide sequence determination of the entire mec DNA of pre-methicillin-resistant Staphylococcus aureus N315. Antimicrob Agents Chemother. 1999;43(6):1449-1458. 
17. Wu Z, Li F, Liu D, Xue H, Zhao X. Novel Type XIl staphylococcal cassette chromosome mec harboring a new cassette chromosome recombinase, CcrC2. Antimicrob Agents Chemother. 2015;59(12):7597601.

18. Baig S, Johannesen TB, Overballe-Petersen S, Larsen J, Larsen AR, Stegger M. Novel SCCmec type XIII (9A) identified in an ST152 methicillin-resistant Staphylococcus aureus. Infect Genet Evol. 2018;61:74-6.

19. Urushibara N, Aung MS, Kawaguchiya M, Kobayashi N. Novel staphylococcal cassette chromosome mec (SCCmec) type XIV (5A) and a truncated SCCmec element in SCC composite islands carrying speG in ST5 MRSA in Japan. J Antimicrob Chemother. 2020;75(1):46-50.

20. Hiramatsu K, Kondo N, Ito T. Genetic basis for molecular epidemiology of MRSA. J Infect Chemother. 1996;2(3):117-29.

21. Hiramatsu K, Cui L, Kuroda M, Ito T. The emergence and evolution of methicillin-resistant Staphylococcus aureus. Trends Microbiol. 2001;9(10):486-93.

22. Kondo Y, Ito T, Ma XX, Watanabe S, Kreiswirth BN, Etienne J, et al. Combination of multiplex PCRs for staphylococcal cassette chromosome mec type assignment: rapid identification system for $m e c, c c r$, and major differences in junkyard regions. Antimicrob Agents Chemother. 2007;51(1):264-74.

23. Oliveira DC, de Lencastre H. Multiplex PCR strategy for rapid identification of structural types and variants of the mec element in methicillin-resistant Staphylococcus aureus. Antimicrob Agents Chemother. 2002;46(7):2155-61.

24. Larsen MV, Cosentino S, Rasmussen S, Friis C, Hasman H, Marvig RL, et al. Multilocus sequence typing of total-genome-sequenced bacteria. J Clin Microbiol. 2012;50(4):1355-61.

25. Li S, Sun S, Yang C, Chen H, Yin Y, Li H, et al. The changing pattern of population structure of Staphylococcus aureus from bacteremia in China from 2013 to 2016: ST239-030-MRSA Replaced by ST59-t437. Front Microbiol. 2018;9:332.

26. Matsumoto K, Kudo K, Uzuka Y, Watanabe K, Nagatake T, Rikitomi N, et al. The pathogenic strains of Staphylococcus aureus lately isolated in Japan. Chemother. 1984;32(8):527-33.

27. Sasaki T, Tsubakishita S, Tanaka Y, Sakusabe A, Ohtsuka M, Hirotaki S, et al. Multiplex-PCR method for species identification of coagulase-positive staphylococci. J Clin Microbiol. 2010;48(3):765-9.

28. Clinical and Laboratory Standards Institute. Performance standards for antimicrobial susceptibility testing. In: Twenty-second informational supplement M100-S22; Vol.32 No.3; Clinical and Laboratory Standards Institute, Waine, PA, USA; 2012.

29. Hiramatsu K, Kihara H, Yokota T. Analysis of borderline-resistant strains of methicillin-resistant Staphylococcus aureus using polymerase chain reaction. Microbiol Immunol. 1992;36(5):445-53.

30. Murakami K, Minamide W, Wada K, Nakamura E, Teraoka H, Watanabe S. Identification of methicillinresistant strains of staphylococci by polymerase chain reaction. J Clin Microbiol. 1991;29(10):22404.

31. Enright MC, Day NP, Davies CE, Peacock SJ, Spratt BG. Multilocus sequence typing for characterization of methicillin-resistant and methicillin-susceptible clones of Staphylococcus aureus. 
J Clin Microbiol. 2000;38(3):1008-15.

32. Harmsen $D$, Claus $H$, Witte W, Rothganger $J$, Claus $H$, Turnwald $D$, et al. Typing of methicillin-resistant Staphylococcus aureus in a university hospital setting by using novel software for spa repeat determination and database management. J Clin Microbiol. 2003;41(12):5442-8.

33. Strommenger B, Braulke C, Heuck D, Schmidt C, Pasemann B, Nübel U, et al. spa typing of Staphylococcus aureus as a frontline tool in epidemiological typing. Journal of clinical microbiology 2008;46(2):574-581.

34. Becker K, Roth R, Peters G. Rapid and specific detection of toxigenic Staphylococcus aureus: use of two multiplex PCR enzyme immunoassays for amplification and hybridization of staphylococcal enterotoxin genes, exfoliative toxin genes, and toxic shock syndrome toxin 1 gene. J Clin Microbiol. 1998;36(9):2548-53.

35. Lina G, Piémont Y, Godail-Gamot F, Bes M, Peter MO, Gauduchon V, et al. Involvement of PantonValentine leukocidin-producing Staphylococcus aureus in primary skin infections and pneumonia. Clin Infect Dis. 1999;29(5):1128-32.

36. Monday SR, Bohach GA. Use of multiplex PCR to detect classical and newly described pyrogenic toxin genes in staphylococcal isolates. J Clin Microbiol. 1999;37(10):3411-4.

37. Joensen KG, Scheutz F, Lund O, Hasman H, Kaas RS, Nielsen EM, et al. Real-time whole-genome sequencing for routine typing, surveillance, and outbreak detection of verotoxigenic Escherichia coli. J Clin Microbiol. 2014;52(5):1501-10.

38. Zankari E, Hasman H, Cosentino S, Vestergaard M, Rasmussen S, Lund O, et al. Identification of acquired antimicrobial resistance genes. J Antimicrob Chemother. 2012;67(11):2640-4.

39. Bartels MD, Petersen A, Worning P, Nielsen JB, Larner-Svensson H, Johansen HK, et al. Comparing whole-genome sequencing with Sanger sequencing for spa typing of methicillin-resistant Staphylococcus aureus. J Clin Microbiol. 2014;52(12):4305-8.

40. Kaas RS, Leekitcharoenphon P, Aarestrup FM, Lund O. Solving the problem of comparing whole bacterial genomes across different sequencing platforms. PLoS One. 2014;9(8):e104984.

41. McGavin MJ, Arsic B, Nickerson NN. Evolutionary blueprint for host- and niche-adaptation in Staphylococcus aureus clonal complex CC30. Front Cell Infect Microbiol. 2012;2:48.

42. Tewhey R, Cannavino CR, Leake JA, Bansal V, Topol EJ, Torkamani A, et al. Genetic structure of community acquired methicillin-resistant Staphylococcus aureus USA300. BMC Genom. 2012;13:508.

43. Alam MT, Read TD, Petit RA, Boyle-Vavra S, Miller LG, Eells SJ, et al. Transmission and microevolution of USA300 MRSA in U.S. households: evidence from whole-genome sequencing. mBio. 2015;6(2):e00054.

44. Vogel V, Falquet L, Calderon-Copete SP, Basset P, Blanc DS. Short term evolution of a highly transmissible methicillin-resistant Staphylococcus aureus clone (ST228) in a tertiary care hospital. PLoS One. 2012;7(6):e38969. 
45. Taneike I, Otsuka T, Dohmae S, Saito K, Ozaki K, Takano M, et al. Molecular nature of methicillinresistant Staphylococcus aureus derived from explosive nosocomial outbreaks of the 1980s in Japan. FEBS Lett. 2006;580(9):2323-34.

46. Gillespie WA, Alder VG. Control of an outbreak of staphylococcal infection in a hospital. Lancet. 1957;272(6969):632-4.

47. Rountree PM, Rheuben J. Penicillin-resistant Staphylococci in the general population. Med J Aust. 1956;43(10):399-402.

48. Rountree PM, Beard MA. Further observations on infection with phage type 80 staphylococci in Australia. Med J Aust. 1958;45(24):789-95.

49. Shaffer TE, Sylvester RF Jr, Baldwin JN, Rheins MS. Staphylococcal infections in newborn infants. II. Report of 19 epidemics caused by an identical strain of staphylococcus pyogenes. Am J Public Health Nations Health. 1957;47(8):990-4.

50. Bynoe ET, Elder RH, Comtois RD. Phage-typing and antibiotic-resistance of staphylococci isolated in a general hospital. Can J Microbiol. 1956;2(3):346-58.

51. Jessen O, Rosendal K, Bulow P, Faber V, Eriksen KR. Changing staphylococci and staphylococcal infections. A ten-year study of bacteria and cases of bacteremia. N Engl J Med. 1969;281(12):62735.

52. Imai D, Ma XX, Yanagawa H, Ito T, Hiramatsu K, Kurosawa H. Characteristics of MRSA strains isolated in Japan in 1999. Juntendo Medical Journal. 2003;49(3):343-54.

53. Ito T, Katayama Y, Asada K, Mori N, Tsutsumimoto K, Tiensasitorn C, et al. Structural comparison of three types of staphylococcal cassette chromosome mec integrated in the chromosome in methicillin-resistant Staphylococcus aureus. Antimicrob Agents Chemother. 2001;45(5):1323-36.

54. Aiba Y, Katayama Y, Hishinuma T, Murakami-Kuroda H, Cui L, Hiramatsu K. Mutation of RNA polymerase beta-subunit gene promotes heterogeneous-to-homogeneous conversion of beta-lactam resistance in methicillin-resistant Staphylococcus aureus. Antimicrob Agents Chemother. 2013;57(10):4861-71.

55. Kondo N, Kuwahara-Arai K, Kuroda-Murakami H, Tateda-Suzuki E, Hiramatsu K. Eagle-type methicillin resistance: new phenotype of high methicillin resistance under mec regulator gene control. Antimicrob Agents Chemother. 2001;45(3):815-24.

56. Kuroda M, Kuroda H, Oshima T, Takeuchi F, Mori H, Hiramatsu K. Two-component system VraSR positively modulates the regulation of cell-wall biosynthesis pathway in Staphylococcus aureus. Mol Microbiol. 2003;49(3):807-21.

57. Kuroda M, Kuwahara-Arai K, Hiramatsu K. Identification of the up- and down-regulated genes in vancomycin-resistant Staphylococcus aureus strains Mu3 and Mu50 by cDNA differential hybridization method. Biochem Biophys Res Commun. 2000;269(2):485-90.

58. Yamakawa J, Aminaka M, Okuzumi K, Kobayashi H, Katayama Y, Kondo S, et al. Heterogeneously vancomycin-intermediate Staphylococcus aureus (hVISA) emerged before the clinical introduction of vancomycin in Japan: a retrospective study. J Infect Chemother. 2012;18(3):406-9. 
59. Dominguez MA, de Lencastre H, Linares J, Tomasz A. Spread and maintenance of a dominant methicillin-resistant Staphylococcus aureus (MRSA) clone during an outbreak of MRSA disease in a Spanish hospital. J Clin Microbiol. 1994;32(9):2081-7.

60. Mato R, Santos Sanches I, Venditti M, Platt DJ, Brown A, Chung M, et al. Spread of the multiresistant Iberian clone of methicillin-resistant Staphylococcus aureus (MRSA) to Italy and Scotland. Microb Drug Resist. 1998;4(2):107-12.

61. Roberts RB, Tennenberg AM, Eisner W, Hargrave J, Drusin LM, Yurt R, et al. Outbreak in a New York City teaching hospital burn center caused by the Iberian epidemic clone of MRSA. Microb Drug Resist. 1998;4(3):175-83.

62. Sanches IS, Ramirez M, Troni H, Abecassis M, Padua M, Tomasz A, et al. Evidence for the geographic spread of a methicillin-resistant Staphylococcus aureus clone between Portugal and Spain. J Clin Microbiol. 1995;33(5):1243-6.

63. Lakhundi S, Zhang K. Methicillin-Resistant Staphylococcus aureus: Molecular Characterization, Evolution, and Epidemiology. Clin Microbiol Rev. 2018;31(4).

64. Rodriguez-Noriega E, Seas C, Guzman-Blanco M, Mejia C, Alvarez C, Bavestrello L, et al. Evolution of methicillin-resistant Staphylococcus aureus clones in Latin America. Int J Infect Dis. 2010;14(7):e560-6.

65. Aires De Sousa M, Miragaia M, Sanches IS, Avila S, Adamson I, Casagrande ST, et al. Three-year assessment of methicillin-resistant Staphylococcus aureus clones in Latin America from 1996 to 1998. J Clin Microbiol. 2001;39(6):2197-205.

66. Sola C, Cortes P, Saka HA, Vindel A, Bocco JL. Evolution and molecular characterization of methicillin-resistant Staphylococcus aureus epidemic and sporadic clones in Cordoba, Argentina. J Clin Microbiol. 2006;44(1):192-200.

67. Becker AP, Santos O, Castrucci FM, Dias C, D'Azevedo PA. First report of methicillin-resistant Staphylococcus aureus Cordobes/Chilean clone involved in nosocomial infections in Brazil. Epidemiol Infect. 2012;140(8):1372-5.

68. Teixeira MM, Araujo MC, Silva-Carvalho MC, Beltrame CO, Oliveira CC, Figueiredo AM, et al. Emergence of clonal complex 5 (CC5) methicillin-resistant Staphylococcus aureus (MRSA) isolates susceptible to trimethoprim-sulfamethoxazole in a Brazilian hospital. Braz J Med Biol Res. 2012;45(7):637-43.

69. Kuroda M, Ohta T, Uchiyama I, Baba T, Yuzawa H, Kobayashi I, et al. Whole genome sequencing of meticillin-resistant Staphylococcus aureus. Lancet. 2001;357(9264):1225-40.

70. Harada D, Nakaminami H, Miyajima E, Sugiyama T, Sasai N, Kitamura Y, et al. Change in genotype of methicillin-resistant Staphylococcus aureus (MRSA) affects the antibiogram of hospital-acquired MRSA. J Infect Chemother. 2018;24(7):563-9.

\section{Additional Files}


Explanation of the additional files

\section{Additional_file_2.xls.}

Table 2. Genetic characterization of the strains in this study.

${ }^{a}$ MLST, Multilocus sequence typing; SCCmec, Staphylococcal cassette chromosome mec, MRSA, methicillin-resistant Staphylococcus aureus; MSSA, methicillin-susceptible Staphylococcus aureus; NT, non-typable.

\section{Additional_file_3.xls.}

Table 3. Minimum inhibitory concentrations of antimicrobial agents against mecA-positive Staphylcoccus aureus and mecAnegative methicillin-resistant Staphylococcus aureus strains in this study by SCCmec type.

${ }^{\text {a }} \mathrm{MIC}_{50} / \mathrm{MIC}_{90}$, MIC required to inhibit the growth of $50 \%$ or $90 \%$ of the strains, respectively; Bold letters mean MIC greater than the breakpoints.

b MICs of oxacilllin and cefoxitin were determined by the agar dilution method; all other MICs were determined by the broth microdilution method.

${ }^{c}$ MRSA, methicillin-resistant Staphylococcus aureus; $\mathrm{SCC} m e c$, Staphylococcal cassette chromosome $m e c$, MIC, minimum inhibitory concentration.

\section{Figures}




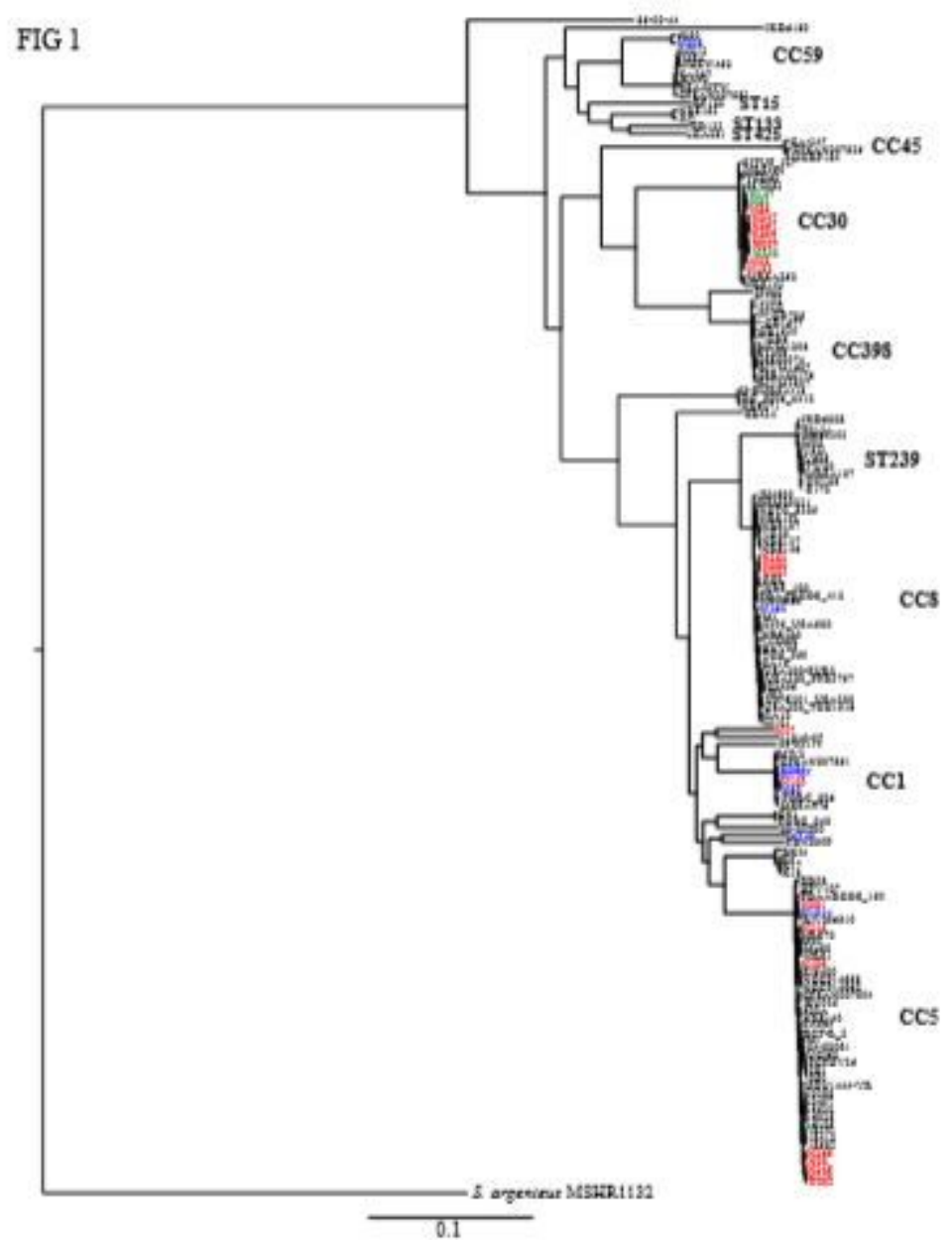

\section{Figure 1}

Phylogenetic tree of the strains in this study and other reference genomes. Phylogenetic tree based on whole-genome SNPs of reference genomes (black-colored font) including mecA-positive MRSA (redcolored font), mecA-negative MRSA (blue-colored font), and mecA-positive MSSA (green-colored font) isolated in this study. NJ tree was constructed by alignment of 41,910 SNP sites. Staphylococcus argenteus MSHR1132 was used as the outgroup. CC, clonal complex; MRSA, methicillin-resistant Staphylococcus aureus; MSSA, methicillin-susceptible Staphylococcus aureus; NJ, neighbor-joining; SNPs, single nucleotide polymorphisms; ST, sequence type. 


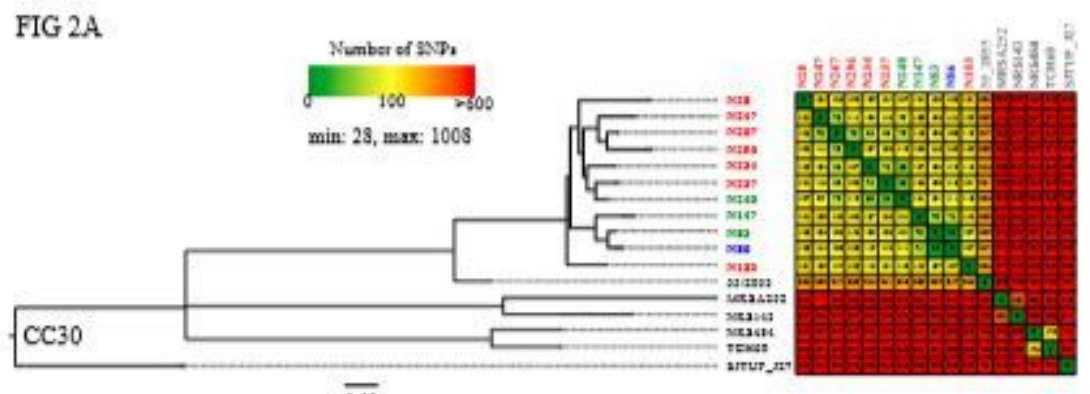

$\overline{0.02}$

FIG 2B

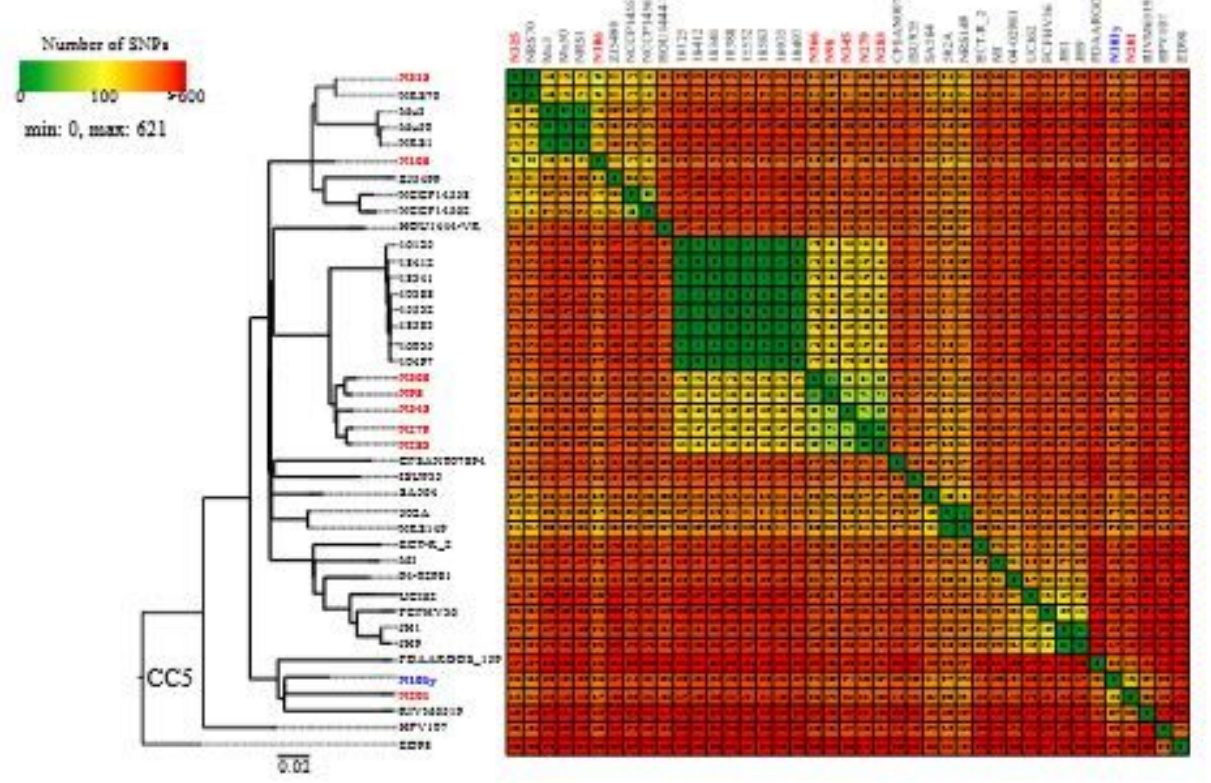

\section{Figure 2}

Phylogenetic tree of the strains in CC30 and CC5. Phylogenetic tree based on whole-genome SNPs in complete genome sequenced-reference strains (black-colored font), mecA-positive MRSA (red-colored font), mecA-negative MRSA (blue-colored font), and mecA-positive MSSA (green-colored font) isolated in this study. The numbers of inter-strain SNP differences were visualized in a red-yellow-green gradient with red indicating the top score $(>600)$ and green indicating the bottom score (0). Phylogenetic tree based on whole-genome SNPs in CC30 strains. Staphylococcus aureus strain SJTUF_J27 was used as the outgroup. NJ tree was constructed by the alignment of 2,353 SNP sites. Phylogenetic tree based on whole-genome SNPs in CC5 strains. Staphylococcus aureus strain ED98 was used as the outgroup. NJ tree was constructed by the alignment of 3,684 SNP sites. NJ, neighbor-joining; SNPs, single nucleotide polymorphisms.

\section{Supplementary Files}

This is a list of supplementary files associated with this preprint. Click to download.

- Additionalfile3.xlsx

- Additionalfile2.xlsx 